\title{
Aufbau eines Markenimages im Krankenhaus „Innen gestalten, um nach außen zu wirken“
}

Mit dem Ansatz „Innen gestalten, um nach außen zu wirken“ gestaltet Ruhl Consulting fachabteilungsspezifisch Strukturen entlang des Behandlungsprozesses, die eine positive Wirkkraft auf Patienten, Einweiser, Mitarbeiter und Träger haben. Dabei stellen Stationen die zentralen leistungserbringenden Einheiten mit direktem Patientenkontakt dar. Eine nachhaltige Neuorganisation der Stationsabläufe und Kommunikationsstrukturen durch die Einführung integrativer Stationskonzepte scheint ein erfolgswirksamer Weg zu begeisterten Patienten, zur Markenbildung und damit zur Bindung der Anspruchsgruppen an das Krankenhaus zu sein.

Es ist schon ganz schön bitter, als Chefarzt, Stationsleitung oder Qualitätsmanager die Fülle an Patientenbeschwerden lesen zu müssen, die im Laufe der Wochen und Monate im Briefkasten des Beschwerdemanagements zu finden sind. Dabei nimmt doch jeder Mitarbeiter noch mehr in Zeiten des heute herrschenden Fachkräftemangels - täglich große Anstrengungen auf sich, seine Arbeit so gut wie möglich zu machen.

Doch bei allen Kritikpunkten, die in einer solchen Patientenbeschwerde aufgegriffen werden, ist sie doch als positiv zu werten. Warum? Weil der Patient noch mit Ihnen spricht! Er bringt Ihnen gegenüber seinen Unmut zum Ausdruck und gibt Ihnen somit die Möglichkeit, seine Kritik konstruktiv aufzugreifen und im Sinne einer kontinuierlichen Verbesserung zu nutzen! Erst wenn der Patient mit seinen Anliegen bei Ihnen kein Gehör mehr findet, wird er seiner Botschaft anderweitig Luft machen, sei es in seinem privaten Umfeld oder - noch schlimmer - in aller Öffentlichkeit, was im Zeitalter des Internets und der Internetforen über- haupt kein Problem mehr ist. Eine solche schlechte Mund-zu-Mund-Propaganda ist das Schlimmste, was dem Ruf Ihres Hauses passieren kann.

\section{Von der schlechten Mund-zu- Mund-Propaganda zur Weiter- empfehlung}

Eigentlich erstrebenswert wäre doch die Situation, dass Patienten begeistert über die Behandlung und den persönlichen Umgang das Krankenhaus verlassen. Dass sie positive Botschaften über Ihr Haus verbreiten und damit fast schon missionarisch Werbung für Sie machen. Doch wie kann dies gelingen?

Ausschlaggebend für die Patienten- bzw. Kundenzufriedenheit und damit für die positive Rückmeldung durch den Kunden, ist die wahrgenommene Qualität. Wahrgenommene Qualität entsteht dabei durch einen permanenten Abgleich zwischen der vom Kunden erwarteten und der vorgefundenen Qualität.

Klaus Kobjoll unterscheidet für den Dienstleistungsbereich 4 Säulen der Servicequalität (Abb. 1).

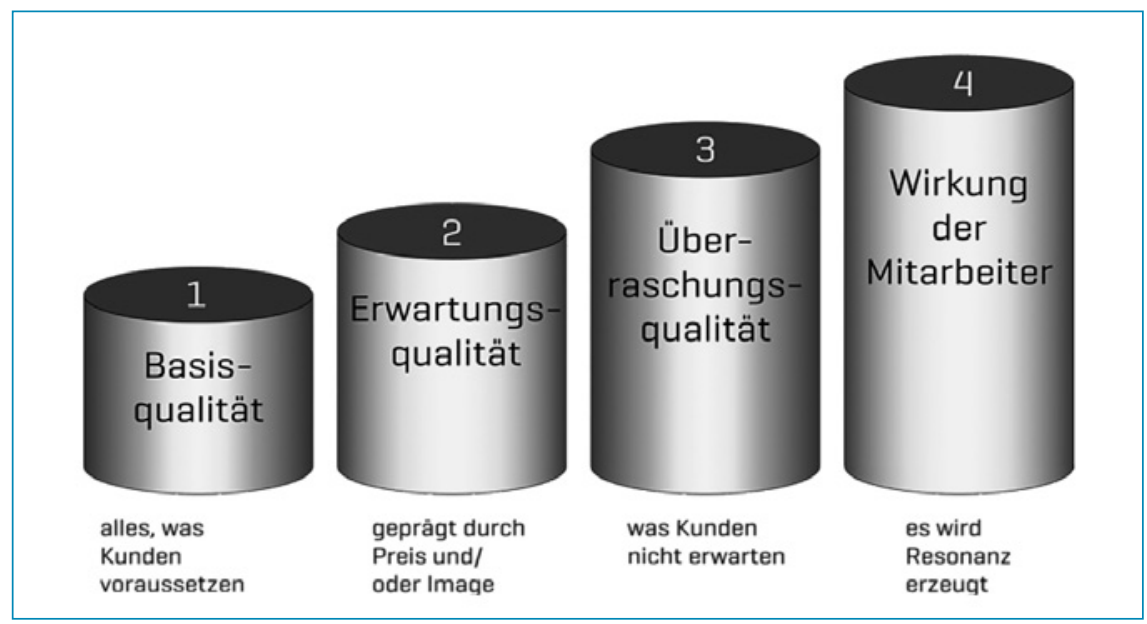

Abb. 1 Die 4 Säulen der Qualität (in Anlehnung an [1]).

\section{Basisqualität}

Basisqualität ist das, was der Kunde beim Kauf einer Dienstleistung mindestens voraussetzt. Aufgrund des technischen Fortschritts steigen die Anforderungen des Kunden an die Basisqualität stetig.

\section{Erwartungsqualität}

Je nach Image und Werbeversprechen erwartet der Kunde neben dem Basisangebot weiteren Service. Erwartungsqualität entsteht also durch die Geister, die Sie als Unternehmen selbst rufen.

Werden Basis- und Erwartungsqualität nicht erfüllt, werden die Kunden das Dienstleistungsangebot nicht wahrnehmen und zur Konkurrenz abwandern.

\section{Überraschungsqualität}

Wie es der Name schon sagt, besteht Überraschungsqualität aus Eigenschaften, die der Kunde nicht erwartet, jedoch positiv überrascht annimmt. Es sind stets Eigenschaften, die seine individuellen Interessen wie Wünsche „von den Augen“ abgelesen erfüllen.

\section{Wirkung der Mitarbeiter}

Durch die Wirkung der Mitarbeiter, ihre Begeisterung und Kommunikationsfähigkeit entsteht Beziehung und Resonanz und so eine emotionale Bindung des Kunden an das Produkt.

Überraschungsqualität und Wirkung der Mitarbeiter sind somit ausschlaggebend dafür, sich von anderen Unternehmen abzuheben, neue Kunden zu gewinnen und dauerhaft zu halten.

\section{Was macht Qualität in der} Patientenversorgung aus?

Was heißt das nun übertragen auf die Situation im Krankenhaus? Was macht Qualität in der Patientenversorgung aus? In Deutschland sind wir stolz auf eine Behandlung von Patienten im Krankenhaus, die auf medizinisch und technisch höchstem Niveau erfolgt. Dennoch sind die Patienten unzufrieden. Patientenbeschwerden, denen wir im Rahmen unserer Projekte begegnet sind, beinhalten in den meisten Fällen Beschwerden über die Organisation der Abläufe (Wartezeiten, unabgestimmte Prozesse auf Station und an 
den Schnittstellen) sowie Beschwerden über die Kommunikation der Mitarbeiter (Zeitmangel, fehlendes Einfühlungsvermögen sowie mangelnde Fähigkeit zum Deeskalieren).

Übertragen auf die 4 Säulen der Qualität heißt das Folgendes:

- Die Basisqualität im Krankenhaus besteht aus einer Versorgung auf dem aktuellen Stand von Medizin und Technik und dem nötigen organisatorischen Know-how. Dies wird von den Patienten mindestens vorausgesetzt, wenn sie ins Krankenhaus eingeliefert werden. Hinzu kommt die Erwartungsqualität, die Sie als Haus über Werbebotschaften in die Öffentlichkeit tragen. Werden Basis- und Erwartungsqualität nicht erfüllt, werden mündige Patienten auf Dauer abwandern.

- Mit der Erfüllung von Basis- und Erwartungsqualität lässt sich Unzufriedenheit vermeiden - von anderen Häusern abheben können Sie sich damit nicht. Dies können Sie lediglich über den Aufbau eines eigenen positiven Markenimages erreichen.

\section{Der Aufbau einer eigenen Marke}

Eine Marke umfasst per definitionem alle Eigenschaften, die Sie von anderen abhebt. Die Theorie der 4 Säulen der Qualität besagt, dass diese Eigenschaften, mit denen individuelle Kundenerwartungen positiv übertroffen werden, in der Überraschungsqualität und in der Wirkung der Mitarbeiter liegen.

Nimmt man die Kritikpunkte der Patientenbeschwerden ernst, kann Überraschungsqualität im Krankenhaus durch verbesserte Behandlungsabläufe erreicht werden. Stellen Sie sich vor, Sie kommen ins Krankenhaus, werden pünktlich aufgenommen, auf Station steht ein Bett für Sie bereit und sowohl Diagnostik- als auch OP-Termin laufen nach einem auf die individuelle Krankengeschichte zugeschnittenen Untersuchungs- und Behandlungsplan pünktlich, koordiniert nacheinander ab - das wäre in der heutigen Gewohnheit doch tatsächlich eine Überraschung!

Eine weitere Möglichkeit, sich von anderen Häusern abzuheben, liegt in der Wirkung der Mitarbeiter. Wenn es Ihren Mitarbeitern gelingt, über empathische Kommunikation und Einfühlungsvermögen während des Klinikaufenthaltes eine persönliche Beziehung zum Patienten aufzubauen, werden Sie sich von vielen

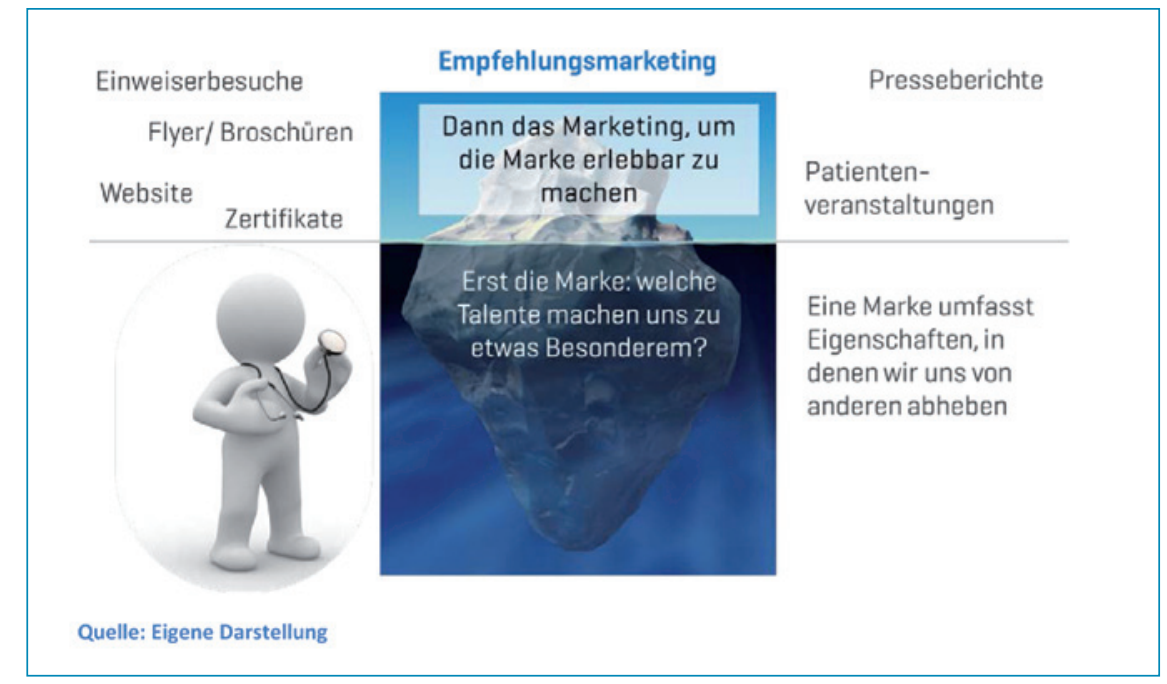

Abb. 2 Der Aufbau einer Marke bis zum Empfehlungsmarketing.

Krankenhäusern unterscheiden.

Wird der Einzelfall immer mehr zur Regel, so lässt sich auf diesem Fundament ein positives Markenimage etablieren. Einen solchen Aufbau einer Marke halten wir für den ersten Schritt hin zu einem erfolgreichen Klinikmarketing. Nur wenn dieser Unterbau steht, kann es gelingen, die Marke mithilfe von Marketinginstrumenten wie Flyern, Broschüren, Einweiserbesuchen, Websites, Zertifikaten, Patientenveranstaltungen bewusst erlebbar zu machen und Empfehlungsmarketing anzuregen (Abb. 2).

\section{Die Station als Ansatzpunkt des Markenerlebens}

Der Aufbau einer eigenen Marke macht vor allem dort Sinn, wo die Marke von allen Anspruchsgruppen, d.h. von Patienten, Angehörigen, Einweisern und Mitarbeitern erlebt werden kann. Im Falle eines mehrtägigen Krankenhausaufenthalts ist dies auf Station.

Im Rahmen unserer zahlreichen Projekte zur Weiterentwicklung des berufsgruppenübergreifenden Stationsmanagements haben wir festgestellt, dass eine nachhaltige Neuorganisation der Stationsabläufe durch interprofessionell und interdisziplinär umgesetzte Stationskonzepte und durch die Verbesserung der Kommunikation ein erfolgswirksamer Weg zur Markenbildung und damit zur Bindung der Anspruchsgruppen an das Krankenhaus ist. Dabei lohnt sich die Anstrengung für das Haus in dreierlei Hinsicht:

- Durch stringente Abläufe und patientennahe Kommunikation steigen Prozesssicherheit und Patientenzufriedenheit
- Durch verbesserte berufsgruppenübergreifende Kommunikation und Auflösen von Bereichsgrenzen entsteht mehr Struktur und Ruhe auf Station und damit steigt die Mitarbeiterzufriedenheit

- Durch die Verringerung von ineffektivem Zeit- und Ressourceneinsatz in den Prozessen erhöht sich die Wirtschaftlichkeit der Patientenbehandlung.

Literatur

1 Kobjoll K. Wa(h)re Herzlichkeit. Orell Fuellki, 2. Auflage 2008: 118

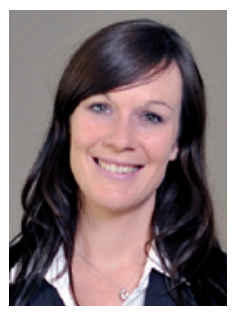

Korrespondenz Katharina Ade Ruhl Consulting AC Harrlachweg 1 68163 Mannheim Tel.: 0621/32886490 E-Mail: info@ruhl-consulting.de www.ruhl-consulting.de

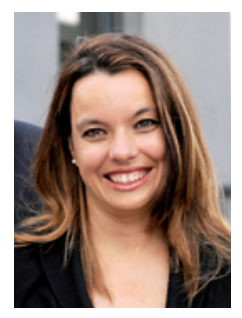

Dr. Elke Eberts Ruhl Consulting AC Harrlachweg 1 68163 Mannheim Tel.: 0621/32886490 E-Mail: info@ruhl-consulting.de www.ruhl-consulting.de 